\title{
RELEASE OF PROGRAM FOR ENHANCED POPULATION HEALTH INFOSTRUCTURE (PEPHI): A REPORT OF RESPONSES TO THE NOVEMBER 2000 DISCUSSION PAPER
}

\section{David Muscatello}

Centre for Epidemiology and Research

NSW Department of Health

The Centre for Epidemiology and Research, NSW Department of Health, has been funded through the National Health Development Fund to enhance access to, and reporting of, population health information for NSW. To assist in developing and refining the work to be conducted under the Program for Enhanced Population Infostructure (PEPHI), we conducted a consultation process commencing in November 2000. The Program for Enhanced Population Health Infostructure (PEPHI): A report of responses to the November 2000 Discussion Paper describes the initiative and the responses received through the consultation process.

In summary, responses to the discussion paper confirm that:

- there is a strong demand for population health information from both within and outside the NSW public health system;

- information that is already available needs to be more visible to potential users;
- printed publications or reports are required for those consumers who have limited computing resources;

- it would be valuable to expand the range of data collections available through the NSW Health Outcomes and Information Statistical Toolkit (HOIST), particularly those that describe social determinants of health;

- making HOIST data more accessible through providing training in its use, and by providing more user-friendly means of obtaining analyses from it, would meet other needs;

- the quality and relevance of PEPHI developments should be monitored to ensure that the Program is meeting its goals.

Copies of the Program for Enhanced Population Health Infostructure (PEPHI): A report of responses to the November 2000 Discussion Paper can be obtained from the Centre for Epidemiology and Research by telephone (02) 93919408 , or from the Department's Web site at: www.health.nsw.gov.au/ public-health/pephi/index.html. 